\title{
The Rise of EBSD for Modern Quantitative Metallography
}

\author{
Scott Sitzman
}

Oxford Instruments America, Inc., Concord, MA, USA

Sorby's pioneering work in characterization began a field of study which would profoundly influence human technological development, affecting all disciplines and industries that benefit from materials properties improvements and the discovery of new materials. Yet, bulk sample metallography remained qualitative throughout most of its history, relying on human visual inspection and interpretation of data recorded on light micrographs. Even as computers and image processing software became available, the microstructure of cubic metals was only visible via indirect contrast mechanisms, such as topography from differential etch rates during preparation. The arrival of the SEM enabled metallography at finer scales, but still relied on inference and indirect recognition of phases, grains and boundaries as differences in topography or density/channeling related changes in backscatter electron intensity.

The physical origin of SEM channeling contrast imaging is subsurface diffraction and backscattering, a phenomenon which would eventually enable direct crystallographic structure and orientation measurement and enter bulk sample metallography into the quantitative realm in the latter part of the $20^{\text {th }}$ century. Just as changes in lattice orientation under a fixed or nearly fixed beam trajectory give changes in backscatter electron yield, changes in beam approach angle for a fixed lattice orientation show systematic variations in backscatter yield, and if mapped as a function of angle of incidence, will describe a Kikuchi-like pattern which may be directly analyzed for orientation, crystal structure and strain. A point analysis technique exploiting this effect, SACP, emerged in the late 1960s [1]. Analysis at finer spatial resolutions are possible using Kikuchi patterns generated from fine, fixed electron probes entering crystallites of fixed orientation, where initially incoherent scattering provides a virtual omnidirectional subsurface electron source for subsequent diffraction and escape, if the distance to the free surface is sufficiently short relative to elastic and inelastic mean free paths. In transmission through a thin sample, this is the basis for TEM-Kikuchi diffraction, and in backscattering from a bulk sample, it is the basis for EBSD in the SEM, in which sample surfaces are highly tilted with respect to the beam and emerging Kikuchi patterns are captured by nearby detectors for analysis. In this way, EBSD collects information about a material by direct determination of phase and orientation from diffraction patterns at individual points, and builds microstructural images by examining how they change with position, at resolutions down to the tens of nanometers. Modern, automated EBSD can perform these diffraction experiments hundreds of times per second over a designed grid, allowing reconstruction of microstructurally representative maps (Fig. 1) and quantitative analyses at a variety of scales, including for grain size, grain shape, grain boundary character, preferred crystallographic orientation, strain visualization, phase distribution, and inter-phase orientation relationships.

Kikuchi patterns in transmission and reflection were first described by their namesake S. Kikuchi with S. Nishikawa (Inst. of Phys. and Chem. Res., Tokyo) in 1928, the latter work on the cleavage face of a piece of calcite, using a gas discharge cylinder and electron-sensitive film [2]. This was followed for the next several decades by other similar work, in which higher quality patterns were collected and more evidence of their physical origin was studied. None of these analyses used micro-electron probes, which weren't widely available for bulk samples until the commercialization of the SEM in 1965. In 1973, J. Venables and C. Harland (U. of Sussex) first observed Kikuchi patterns in an SEM [3], and in the years 
following performed related analyses, including at submicron scales, all using photographs of the phosphor screen collected from outside the SEM chamber. D. Dingley (U. of Bristol) began improving the technique in earnest throughout the 1980s, introducing the use of high sensitivity video cameras, graphical importation to a computer, online computer-assisted indexing software, and the first commercialization [4]. In 1989, N.-H. Schmidt and N. Olesen (Risø Nat'1 Lab \& U. of Aarhus) reduced manual input to simple band position marking [5], removing the requirement of user recognition of zone axis or band identity, in turn enabling automation of this step in 1992 by N. Krieger-Lassen (Risø Nat'l Lab), who employed a Hough image transform and butterfly filter for band detection [6]. In 1993, B. Adams, S. Wright, and K. Kunze (Brigham Young U., Los Alamos Nat'l Lab \& Yale U.) described [7] then commercialized the first fully automated EBSD system, including integrated SEM/stage control, followed by N.-H. Schmidt later that year and others soon after. Also in 1993, J. Michael and R. Goehner (Sandia Nat'l Labs) introduced a true EBSD-based phase ID method, using EDS data and calculated primitive unit cell volume for initial phase database filtering, followed by EBSD indexing [8].

Developments since the early 1990s expanded the versatility of the technique for application across a wide range of materials and scales, including combined beam-stage scanning, the simultaneous collection of EDS data via a coordinated EDS detector and system, and the development of powerful post-acquisition analysis software. In the 2000s, notable developments included high speed detectors capable of acquisition rates in the many hundreds of points per second, dynamic drift correction, 3-D EBSD on FIB-SEMs, in situ heating and deformation experiments, and demonstration of the first highly realistic dynamical EBSD-Kikuchi pattern simulations [9]. Tangential techniques have emerged as well, for example Kikuchi pattern image cross-correlation for ultra-high sensitivity strain analysis [10], and the capture and analysis of transmitted Kikuchi patterns from thin samples in the SEM using conventional EBSD hardware and software for markedly improved spatial resolution $[11,12]$.

References:

[1] D. Joy et al., Journal of Applied Physics 53 (1982) p. 81-122

[2] S. Nishikawa and S. Kikuchi, Nature 22 (1928) p. 726

[3] J. Venables and C. Harland, Philosophical Magazine 27 (1973) p. 1193-1200

[4] D. Dingley, in "Electron Backscatter Diffraction in Materials Science, $1^{\text {st }}$ Ed., eds. A. Schwartz, M. Kumar, D. Field and B. Adams (Kluwer Academic/Plenum Publishers, New York) p. 1-18

[5] N.-H. Schmidt and N. Olesen, Canadian Mineralogist 27 (1989) p. 15-22

[6] N. Krieger-Lassen et al., Scanning Microscopy 6 (1992), p. 115-121

[7] B. Adams et al., Metallurgical Transactions A 24A (1993) p. 819-831

[8] J. Michael and R. Goehner, MSA Bulletin 23 (1993) p. 168

[9] A. Winklemann et al., Ultramicroscopy 107 (2007) p. 414-421

[10] A. Wilkinson et al., Ultramicroscopy 106 (2006) p. 307-313

[11] R. Keller and R. Geiss, Journal of Microscopy 245 (2011) p. 245-251

[12] P. Trimby, Ultramicroscopy 120 (2012) p. 16-24
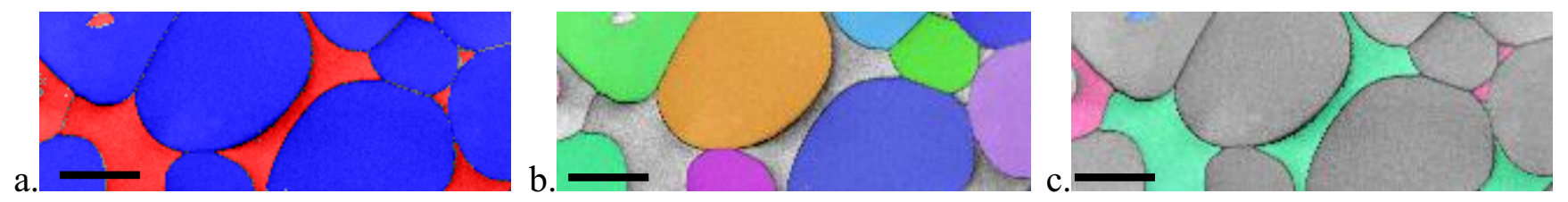

Figure 1. Example portions of EBSD maps from a W-hardened alloy. (a) Phase, with blue $=\mathrm{W}$, red $=$ $\mathrm{Ni}$; Orientation maps for (b) $\mathrm{W}$ and (c) Ni, based on a surface normal-projected IPF. Scale bar $=10 \mu \mathrm{m}$. 